\title{
Report of the Japan Diabetes Society (JDS)/Japanese Cancer Association (JCA) Joint Committee on Diabetes and Cancer, Second Report
}

\author{
Atsushi Goto $^{1} \cdot$ Hiroshi Noto ${ }^{2} \cdot$ Mitsuhiko Noda $^{3} \cdot$ Kohjiro Ueki $^{4} \cdot$ Masato Kasuga $^{5}$. \\ Naoko Tajima ${ }^{6} \cdot K_{\text {Ken }}$ Ohashi $^{7} \cdot$ Ryuichi Sakai $^{8} \cdot$ Shoichiro Tsugane9 ${ }^{9}$ \\ Nobuyuki Hamajima $^{10} \cdot$ Kazuo Tajima $^{11} \cdot$ Kohzoh Imai $^{12} \cdot$ Hitoshi Nakagama $^{13}$
}

Received: 10 December 2015/Published online: 7 March 2016

(C) The Japan Diabetes Society and the Japanese Cancer Association 2016

\begin{abstract}
The Japan Diabetes Society (JDS)/Japanese Cancer Association (JCA) Joint Committee on Diabetes and Cancer published its first report in July 2013 on the epidemiological assessment of the associations of diabetes with cancer risk/prognosis, the common risk factors for diabetes and cancer, and cancer risk associated with diabetes treatment The JDS/JCA Joint Committee continued its work to assess the role of glycemic control in the development of cancer in patients with diabetes. This review shows that high-quality evidence examining the association between glycemic control and cancer risk is lacking.
\end{abstract}

In 2014, the Japan Diabetes Society (JDS) and the Japanese Cancer Association (JCA) restarted the JDS/JCA Joint Committee on Diabetes and Cancer, which published the second committee report in Japanese [1]. This is the English version of that report.

This article has been jointly published in Diabetology International and Cancer Science (doi:10.1111/cas.12889) by the Japan Diabetes Society and the Japanese Cancer Association.

Kohjiro Ueki

ueki-tky@umin.net

Tokyo Women's Medical University, Tokyo, Japan

2 St. Luke's International Hospital, Tokyo, Japan

3 Department of Endocrinology and Diabetes, Saitama Medical University, Saitama, Japan

4 Department of Diabetes and Metabolic Diseases, Graduate School of Medicine, University of Tokyo, Tokyo, Japan

5 National Center for Global Health and Medicine, Tokyo, Japan

6 Jikei University School of Medicine, Tokyo, Japan

7 Department of General Internal Medicine, Dentistry, and Oncologic Emergencies, National Cancer Center Hospital, Tokyo, Japan
Keywords Diabetes - Cancer - Glucose control - HbA1c · Randomized controlled trials · Observational studies

\section{Background}

The Japan Diabetes Society (JDS)/Japanese Cancer Association (JCA) Joint Committee on Diabetes and Cancer (hereafter, JDS/JCA Joint Committee) published its first report in July 2013 on the epidemiological assessment of the associations of diabetes with cancer risk/prognosis, the common risk factors for diabetes and cancer, and cancer risk associated with diabetes treatment [2]. The JDS/JCA Joint Committee continued its work, while focusing on glycemic control and cancer risk in patients with diabetes; this work was followed by its sixth and seventh meetings held on April 2, 2014 and September 2, 2015, respectively.

To date, several meta-analyses and pooled analyses have unequivocally shown that people with diabetes are at a

8 Department of Biochemistry, Kitasato University School of Medicine, Sagamihara, Japan

9 National Cancer Center Research Center for Cancer Prevention and Screening, Tokyo, Japan

10 Department of Healthcare Administration, Nagoya University Graduate School of Medicine, Nagoya, Japan

11 Department of Public Health and Occupational Medicine, Mie University Graduate School of Medicine, Tsu, Japan

12 Center for Antibody and Vaccine Therapy, The Institute of Medical Science, The University of Tokyo, Tokyo, Japan

13 National Cancer Center Research Institute, Tokyo, Japan 
higher risk for pancreatic and hepatic cancer as well as for overall cancer than are those without diabetes [3-5]. On the other hand, whether glycemic control is associated with cancer risk in patients with diabetes has not been sufficiently explored. Thus, the current report of the JDS/JCA Joint Committee intends to provide a summary of the evidence available for an association between glycemic control and cancer risk in patients with diabetes.

\section{Evidence from randomized controlled trials}

The Action in Diabetes and Vascular Disease: Preterax and Diamicron Modified-Release Controlled Evaluation (ADVANCE) study investigators evaluated the effect of intensive glycemic control on cancer risk in patients with diabetes by using data obtained from that study [6]. The ADVANCE study was a large-scale randomized controlled trial conducted in 80 countries worldwide and included, in total, 11,140 patients with type 2 diabetes and a history of a major cardiovascular disease (CVD) or microangiopathy or at least 1 risk factor for CVD [7]. In that study, the subjects were randomly assigned to receive intensive glycemic control with gliclazide and other oral hypoglycemic agents, and the glycemic control target was defined as a glycated hemoglobin (HbA1c) level of $6.5 \%$ or less (intensive therapy group), or to receive conventional glycemic control with standard therapy in each region or country (conventional therapy group). The mean $\mathrm{HbAlc}$ value in the ADVANCE study decreased from $7.5 \%$ at baseline in both the groups to $6.5 \%$ in the intensive therapy group versus $7.3 \%$ in the conventional therapy group after a 5-year follow-up. In addition, intensive glycemic control was prospectively evaluated for its influence on the cancer risk, defined as cancer morbidity or mortality according to the reported adverse events and documented deaths; death from cancer was assessed by an independent assessment committee blinded to the study subject allocation. During the median follow-up of 5 years, cancer events occurred in 363 patients (1.39 cases/100 person-years) in the intensive therapy group versus 337 patients $(1.28$ cases/100 personyears) in the conventional therapy group, and this difference between the groups was not significant [hazard ratio 1.08 ; $95 \%$ confidence interval (CI) $0.93-1.26]$. The cancer events included 41 and 35 deaths from cancer in the intensive and conventional therapy groups, respectively. The allocation sequence was appropriately generated and concealed and patient dropout rate was low in both the intensive and conventional therapy groups ( 7 and 10 patients, respectively); however, the study had limitations, in that the assessors were not blinded to the subject allocation information. Cancer events were not evaluated as the primary outcome measures, and the follow-up was not sufficiently long to draw any conclusions on the cancer risk associated with glycemic control.

To date, a meta-analysis of seven randomized controlled trials, including the ADVANCE study, has been performed to evaluate the cancer risk associated with intensive glycemic control (Table 1) [8]. The United Kingdom Prospective Diabetes Study (UKPDS) 33 [9], UKPDS 34 [10], Action to Control Cardiovascular Risk in Diabetes (ACCORD) study [11], and Veterans Affairs Diabetes Trial (VADT) [12] were included for the analysis of cancer mortality as an outcome measure. The results of the analysis showed that during the follow-up of 3.5-10.7 years, cancer deaths occurred in 222/53,892 person-years receiving intensive glycemic control versus 155/38,743 person-years receiving conventional glycemic control, and the overall risk ratio as estimated by a random-effects model was 1.00 (95\% CI $\left.0.81-1.24 ; \mathrm{I}^{2}=0 \%\right)$. The ADVANCE study [7], PROspective pioglitAzone Clinical Trial In MacroVascular Events (PROACTIVE) study [13], and Rosiglitazone Evaluated for Cardiac Outcomes and Regulation of Glycaemia in Diabetes (RECORD) study

Table 1 Incidence of cancer and cancer deaths in major randomized controlled trials

\begin{tabular}{|c|c|c|c|c|c|c|c|}
\hline & ACCORD & ADVANCE & RECORD & PROACTIVE & UKPDS 33 & UKPDS 34 & $\overline{\text { VADT }}$ \\
\hline $\begin{array}{l}\text { No. of patients on intensive/conventional } \\
\text { therapy }\end{array}$ & $5,128 / 5123$ & $5,645 / 5,038$ & $2,220 / 2,227$ & $2,605 / 2,633$ & $2,729 / 1,138$ & $342 / 411$ & $892 / 899$ \\
\hline Mean age (years) & 62 & 66 & 58 & 62 & 53 & 53 & 60 \\
\hline Duration of diabetes (years) & 10 & 8 & 7 & 8 & $<1$ & $<1$ & 11.5 \\
\hline $\mathrm{HbA} 1 \mathrm{c}(\%)$ at the initiation of therapy & 8.3 & 7.5 & 7.9 & 7.9 & 7.1 & 7.2 & 9.4 \\
\hline $\begin{array}{l}\text { Cancer incidence }(n) \text { in the } \\
\text { intensive/conventional therapy group }\end{array}$ & nd & $119 / 119$ & $126 / 148$ & $112 / 113$ & nd & nd & nd \\
\hline $\begin{array}{l}\text { Cancer mortality }(n) \text { in the } \\
\text { intensive/conventional therapy group }\end{array}$ & $65 / 63$ & nd & nd & nd & $120 / 50$ & $13 / 21$ & $24 / 21$ \\
\hline
\end{tabular}

Adapted from Johnson et al. [8]

nd no data 
[14] were included for the analysis of the incidence of cancer as an outcome measure. The results of the analysis showed that during the follow-up of 2.9-5.5 years, cancer occurred in $357 / 47,924$ person-years receiving intensive glycemic control versus 380/45,009 person-years receiving conventional glycemic control, and the overall risk ratio as estimated by a random-effects model was 0.91 (95\% CI $\left.0.79-1.05 ; \mathrm{I}^{2}=0 \%\right)$. However, this meta-analysis has some limitations, in that it included very few studies and might have been associated with a publication bias. Furthermore, cancer mortality or incidence was not a primary outcome measure in the original studies; the analysis included non-blinded studies; and the follow-up period in the included studies was very short.

Thus, to date, no high-quality randomized controlled trial has been performed to estimate cancer risk associated with glycemic control.

\section{Evidence from observational studies}

In a Hong Kong registry study involving 4,623 patients with diabetes and a mean follow-up of 4.8 years among insulin users and of 6.0 years among non-insulin users, the association of HbA1c level with cancer risk was examined. In that study, the incidence of cancer was defined as hospital admissions according to the International Classification of Diseases, Ninth Revision (ICD-9) (ICD-9 codes 140-208). An examination of these patients showed that the HbA1c level was associated with an increased risk for all cancers (odds ratio for every $1 \%$ increase in the HbA1c level, 1.17; $95 \%$ CI 1.04-1.33) after adjustment for multiple factors, including their age, history of smoking, insulin use, metformin use, serum high-density-lipoprotein (HDL)-cholesterol levels, and serum triglyceride levels [15]. Sex was not included as a covariate in this analysis, because it was not associated with cancer risk in a bivariate analysis $(P>0.3)$.

Subsequently, in a cohort study, 25,476 Swedish patients with type 2 diabetes were followed from 1997-1999 to 2009. In that study, the incidence of cancer was defined according to the cancer registry data (ICD-10 C00-C97, D00-D09, and D37-D48). The data indicated that the hazard ratio (HR) for cancer incidence among patients with an HbA1c level of $>7.5 \%$, as compared to that among patients with an HbA1c level of $\leq 7.5 \%$, was 1.02 (95\% CI 0.95-1.10) after adjustment for such covariates as age, sex, duration of diabetes, and insulin therapy. Furthermore, an HbA1c level of $>7.5 \%$ was not associated with the incidence of any particular cancer [16]. Similar analyses were performed in a cohort study in which 9,486 American patients with type 2 diabetes were followed. In that study, the incidence of cancer was defined according to the electronic medical records or cancer registry data, which showed that $\mathrm{HbA1c}$ values were not associated with the risk of breast cancer or colorectal cancer [17]. Furthermore, patients with lower HbA1c values $(<6.5 \%)$ had a higher risk for prostate cancer than were those with higher HbA1c values $(\geq 7 \%$; HR 1.57; $95 \%$ CI 1.09-2.26) [17]. This inverse association between HbA1c values and prostate cancer risk is consistent with the observation that diabetes is associated with a decreased risk for prostate cancer [3].

In summary, although some observational studies suggest an association between glycemic control and cancer risk, the results are not consistent among the studies, and in general, reports on high-quality epidemiological studies in the literature are scarce.

In this review, we provide an overview of the evidence currently available for the association between glycemic control and cancer risk. Given the paucity of high-quality evidence at present, however, well-designed randomized controlled trials and observational studies are required to explore this issue further.

\section{Compliance with ethical standards}

Conflict of interest Hiroshi Noto received honoraria for manuscripts from Eli Lilly Japan K.K. Mitsuhiko Noda received scholarship grants from Takeda Pharmaceutical Company Limited and AstraZeneca K. K. Kohjiro Ueki received speaker honoraria from MSD K.K, scholarship grants from Takeda Pharmaceutical Company Limited, Daiichi Sankyo Co., Ltd., Ono Pharmaceutical Co., Ltd., Astellas Pharma Inc., Sanofi K. K, Novo Nordisk Pharma Ltd., Eli Lilly Japan K.K, MSD K.K, Boehringer Ingelheim, Mitsubishi-Tanabe Pharma Corporation and courses endowed by MSD K.K, Boehringer Ingelheim and Novo Nordisk Pharma Ltd. Naoko Tajima received speaker honoraria from Astellas Pharma Inc., MSD K.K, Kissei Pharmaceutical Co., Ltd., Takeda Pharmaceutical Company Limited, Eli Lilly Japan K.K, Boehringer Ingelheim and Novo Nordisk Pharma Ltd. Nobuyuki Hamajima received scholarship grants from Sugi Drugstore Co. Ltd. Kohzoh Imai received research grants from DeNA Life Science. Inc. Hitoshi Nakagama received research grants from Shimadzu Corporation and BioMimetics Sympathies Inc. Atsushi Goto, Masato Kasuga, Ken Ohashi, Ryuichi Sakai, Shoichiro Tsugane and Kazuo Tajima declare that they have no conflict of interest.

\section{Appendix}

The following authors are members of the Japan Diabetes Society: Mitsuhiko Noda, Kohjiro Ueki, Masato Kasuga, Naoko Tajima, and Ken Ohashi.

Editorial Collaborators: Atsushi Goto and Hiroshi Noto.

The following authors are members of the Japanese Cancer Association: Ryuichi Sakai, Shoichiro Tsugane, Nobuyuki Hamajima, Kazuo Tajima, Kohzoh Imai, and Hitoshi Nakagama. 


\section{References}

1. Goto A, Noto H, Noda M, et al. Report of the JDS/JCA Joint Committee on Diabetes and Cancer, Second Report. J Japan Diabetes Soc. 2016;59(3):174-77. doi:10.11213/tonyobyo.59.174 (In Japanese)

2. Kasuga M, Ueki K, Tajima N, et al. Report of the JDS/JCA Joint Committee on Diabetes and Cancer. Diabetol Int. 2013;4(2): 81-96.

3. Noto H, Tsujimoto T, Sasazuki T, Noda M. Significantly increased risk of cancer in patients with diabetes mellitus: a systematic review and meta-analysis. Endocr Pract. 2011;17(4): 616-28.

4. Sasazuki S, Charvat H, Hara A, et al. Diabetes mellitus and cancer risk: pooled analysis of eight cohort studies in Japan. Cancer Sci. 2013;104(11):1499-507.

5. Tsilidis KK, Kasimis JC, Lopez DS, Ntzani EE, Ioannidis JP. Type 2 diabetes and cancer: umbrella review of meta-analyses of observational studies. BMJ. 2015;350:g7607.

6. Stefansdottir G, Zoungas S, Chalmers J, et al. Intensive glucose control and risk of cancer in patients with type 2 diabetes. Diabetologia. 2011;54(7):1608-14.

7. Advance Collaborative Group. Intensive blood glucose control and vascular outcomes in patients with type 2 diabetes. N Engl J Med. 2008;358(24):2560-72.

8. Johnson JA, Bowker SL. Intensive glycaemic control and cancer risk in type 2 diabetes: a meta-analysis of major trials. Diabetologia. 2011;54(1):25-31.

9. UK Prospective Diabetes Study (UKPDS) Group. Intensive bloodglucose control with sulphonylureas or insulin compared with conventional treatment and risk of complications in patients with type 2 diabetes (UKPDS 33. Lancet. 1998;352(9131):837-53.
10. UK Prospective Diabetes Study. UKPDS) Group. Effect of intensive blood-glucose control with metformin on complications in overweight patients with type 2 diabetes (UKPDS 34. Lancet. 1998;352(9131):854-65.

11. The Action to Control Cardiovascular Risk in Diabetes Study Group. Effects of intensive glucose lowering in type 2 diabetes. N Engl J Med. 2008;358(24):2545-59.

12. Duckworth W, Abraira C, Moritz T, et al. Glucose control and vascular complications in veterrans with type 2 diabetes. $\mathrm{N}$ Engl $\mathrm{J}$ Med. 2009;360(2):129-39.

13. Dormandy JA, Charbonnel B, Eckland DJ, et al. Secondary prevention of macrovascular events in patients with type 2 diabetes in the PROactive Study (PROspective pioglitAzone Clinical Trial In macroVascular Events): a randomised controlled trial. Lancet. 2005;366(9493):1279-89.

14. Home PD, Pocock SJ, Beck-Nielsen H, et al. Rosiglitazone evaluated for cardiovascular outcomes in oral agent combination therapy for type 2 diabetes (RECORD): a multicentre, randomised, open-label trial. Lancet. 2009;373(9681):2125-35.

15. Yang X, Ko GT, So WY, et al. Associations of hyperglycemia and insulin usage with the risk of cancer in type 2 diabetes: the Hong Kong diabetes registry. Diabetes. 2010;59(5):1254-60.

16. Miao Jonasson J, Cederholm J, Eliasson B, Zethelius B, EegOlofsson K, Gudbjornsdottir S. HbA1C and cancer risk in patients with type 2 diabetes-a nationwide population-based prospective cohort study in Sweden. PLoS One. 2012;7(6): e38784.

17. Onitilo AA, Stankowski RV, Berg RL, et al. Type 2 diabetes mellitus, glycemic control, and cancer risk. Eur J Cancer Prev. 2014;23(2):134-40. 